\title{
Apple Contains Receptor-like Genes Homologous to the Cladosporium fulvum Resistance Gene Family of Tomato with a Cluster of Genes Cosegregating with Vf Apple Scab Resistance
}

\author{
Boris A. Vinatzer,$^{1 *}$ Andrea Patocchi ${ }^{2 *}$ Luca Gianfranceschi, ${ }^{2}$ Stefano Tartarini, ${ }^{1}$ Hong-Bin Zhang, ${ }^{3}$ \\ Cesare Gessler, ${ }^{2}$ and Silviero Sansavini ${ }^{1}$ \\ ${ }^{1}$ Dipartimento di Colture Arboree, Via Filippo Re 6, University of Bologna, 40126 Bologna, Italy; ${ }^{2}$ Plant \\ Pathology Group, Institute of Plant Sciences, Swiss Federal Institute of Technology, Univerisitaetstrasse \\ 2, 8092 Zürich, Switzerland; ${ }^{3}$ Department of Soil and Crop Sciences, Texas A\&M University, College \\ Station 77840, U.S.A. \\ Accepted 20 December 2000.
}

Scab caused by the fungal pathogen Venturia inaequalis is the most common disease of cultivated apple (Malus $x$ domestica Borkh.). Monogenic resistance against scab is found in some small-fruited wild Malus species and has been used in apple breeding for scab resistance. $V f$ resistance of Malus floribunda 821 is the most widely used scab resistance source. Because breeding a high-quality cultivar in perennial fruit trees takes dozens of years, cloning disease resistance genes and using them in the transformation of high-quality apple varieties would be advantageous. We report the identification of a cluster of receptorlike genes with homology to the Cladosporium fulvum ( $C f)$ resistance gene family of tomato on bacterial artificial chromosome clones derived from the $V f$ scab resistance locus. Three members of the cluster were sequenced completely. Similar to the $C f$ gene family of tomato, the deduced amino acid sequences coded by these genes contain an extracellular leucine-rich repeat domain and a transmembrane domain. The transcription of three members of the cluster was determined by reverse transcriptionpolymerase chain reaction to be constitutive, and the transcription and translation start of one member was verified by $5^{\prime}$ rapid amplification of cDNA ends. We discuss the parallels between $C f$ resistance of tomato and $V f$ resistance of apple and the possibility that one of the members of the

Corresponding author: C. Gessler;

E-mail: cesare.gessler@ipw.agrl.ethz.ch

Current address of B. Vinatzer: Department of Molecular Genetics and Cell Biology, University of Chicago, 1103 E. 57th Street, EBC 409, Chicago, IL 60637, U.S.A.; E-mail: bavinatz@ midway.uchicago.edu

Current address of L. Gianfranceschi: Department of Genetics and Biology of Microorganisms, University of Milan, Via Celoria 26, 20133 Milano, Italy; E-mail: luca.gianfranceschi@unimi.it

*The first two authors contributed equally to this work.

Nucleotide and amino acid sequences of HcrVf1, HcrVf2 and HcrVf3 have been submitted to the EMBL database as accession nos. AJ297739, AJ297740, and AJ297741, respectively. gene cluster is the $V f$ gene. $C f$ homologs from other regions of the apple genome also were identified and are likely to present other scab resistance genes.

Additional keyword: durable resistance.

Almost all of our daily fruit supply comes from perennial fruit trees. Consumers demand fruit that is perfectly spotless, but want it produced with the least amount of pesticides. To combine high-quality fruit traits with disease resistance is, therefore, the aim of most fruit-tree breeding programs today (Laurens 1996). As a result of the long juvenile period of perennial fruit trees, breeding programs with this aim take dozens of years to accomplish. $V f$ apple scab resistance is a typical example: $V f$ resistance, inherited as a monogenic trait, has been introgressed in the cultivated apple (Malus $\times$ domestica Borkh.) from the small-fruited, wild Malus spp. accession Malus floribunda 821 through a breeding program that started in 1914 (Crandall 1926), although did not produce the first varieties of acceptable quality and fruit size until 1970 (Dayton et al. 1970).

Another peculiarity of perennial fruit trees is the long replanting cycle of fruit tree orchards (typically 15 to 20 years). Therefore, durable disease resistance is much more important in fruit trees than in any herbaceous crop. Durable disease resistance can be achieved by combining more than one disease resistance gene in a single fruit variety to reduce the risk of a pathogen overcoming a single disease resistance gene. In fact, $V f$ apple scab resistance has been overcome in recent years in many apple-growing areas in Europe (Bénaouf and Parisi 2000; Parisi et al. 1993). Other scab resistance genes are available in wild relatives of apple (Williams and Kuc 1969). Combining these resistance genes with $V f$ resistance would be advantageous. Marker-assisted selection (MAS) with the use of molecular markers for scab resistance could be of great help for such "pyramiding" of scab resistance genes. Because apple is self-incompatible and, therefore, highly heterozygous, all genes will be newly recombined in each gen- 
eration and individuals carrying the resistance genes as well as all other requested quality traits will have to be selected. Even if such an individual is found, it always will be a new variety that has to face the difficult task of replacing the already wellestablished, popular varieties on the market. Transferring multiple scab resistance genes by gene engineering could help to overcome this problem.

Apple scab is caused by the ascomycete Venturia inaequalis, and the interaction between this pathogen and its host has been studied since breeding for apple scab resistance began (Boone 1971; Gessler 1989; Williams and Kuc 1969). Boone (1971) and, more recently, Sierotzki et al. (1994; 1998) and Koch et al. (2000) have shown that every apple variety has resistance genes against all genotypes of $V$. inaequalis, except those missing the corresponding avirulence alleles. In nature, however, the scab inoculum always is a mixture of virulent and avirulent genotypes. Consequently, these resistances are not evident in the field and, for this reason, are called "ephemeral" (Koch et al. 2000). One of these genes, $V g$, determining ephemeral resistance recently has been mapped (Durel, personal communication). Vf scab resistance and other apple scab resistance genes with broad action against most races of $V$. inaequalis also have been mapped (Cheng et al. 1998; Gardiner et al. 1999; Patocchi et al. 1999a). The identified molecular markers of these genes can be used in MAS but, to date, no scab resistance gene has been cloned. We recently reported the construction of an apple bacterial artificial chromosome (BAC) library (Vinatzer et al. 1998), physical mapping of the $V f$ gene region (Patocchi et al. 1999a), and the construction of a BAC contig spanning the entire Vf locus (Patocchi et al. 1999b). The constructed BAC contig is $550 \mathrm{~kb}$ and encompasses the $V f$ region between the molecular markers M18 on one side of the gene and AM19 on the other side. The possible location of the $V f$ gene was restricted in this contig to $350 \mathrm{~kb}$ covered by a minimum of five BAC clones (Patocchi et al. 1999b).

When the first plant disease resistance genes had been cloned in Arabidopsis, flax, and tobacco, a remarkable similarity in the sequences coding for a nucleotide binding siteleucine-rich repeat (NBS-LRR) motifs was observed within some of the genes (Staskawicz et al. 1995). Designing heterologous primers on conserved regions of these genes, resistance gene analogs (RGA) (Leister et al. 1996) that cosegregated with resistance loci were identified in many plant species (Aarts et al. 1998; Kanazin et al. 1996; Leister et al. 1996; Meyers et al. 1998; Yu et al. 1996). This approach did not lead to the identification of NBS-LRR-class RGAs on the $V f$ BAC contig (unpublished data), suggesting a different mechanism to control resistance in the case of $V f$ resistance. In this paper, we report the identification on the $V f \mathrm{BAC}$ contig of a cluster of homologs of the Cladosporium fulvum $(C f)$ resistance genes of tomato (Dixon et al. 1996; Jones et al. 1994; Parniske et al. 1997; Thomas et al. 1997). We show the complete genomic sequence of three genes of the cluster and the analysis of their transcription. We discuss the phenotypic similarities of $C f$ and $V f$ resistance and the possibility that one of the members of the $C f$ homolog gene cluster of the $V f$ region is the $V f$ gene. We also report that other loci in the apple genome hybridize to the $C f$ homologous sequences. These loci could correspond to ephemeral resistance loci or loci for broad-range apple scab resistance genes that could be com- bined with $V f$ resistance to create durable apple scab resistance.

\section{RESULTS}

Identification of a cluster of $\boldsymbol{C} \boldsymbol{f}$ homologs at the $\boldsymbol{V} \boldsymbol{f}$ locus.

Previously we constructed two BAC contigs covering the entire $V f$ locus, one contig consisting of BAC clones derived from the chromosome carrying the allele of the $V f$ locus conferring resistance forming the "resistant" contig and one consisting of BAC clones derived from the chromosome carrying the $v f$ allele forming the "susceptible" contig (Patocchi et al. 1999b). Within the 550-kb resistant contig, we identified five clones (M18-2, M18-5, M18-6, M18-7, and M18-8) that cover $350 \mathrm{~kb}$ and compose the minimum "tiling path" between the two markers closest to $V f$, which are separated from the $V f$ locus by recombinants in our mapping population (Fig. 1A). Their inserts were used as probe to screen 750,000 clones of a cDNA library of leaves of the apple cultivar Florina (inoculated with $V$. inaequalis) in order to identify candidate $V f$ genes. A total of 101 cDNAs hybridizing to the five BAC inserts were identified and sequenced from the poly(A) tail, and 50 different sequences were identified. The corresponding cDNAs also were sequenced from the $5^{\prime}$ end, and the $5^{\prime}$ and $3^{\prime}$ sequences were compared to GenBank. Although they were not full length, three cDNA clones (cDNAs 8-1, 10-5, 15-4), which were very similar to each other, presented homology to the $C f$ resistance genes of tomato (Dixon et al. 1996; Jones et al. 1994; Parniske et al. 1997; Thomas et al. 1997). Their deduced amino acid sequence was $56 \%$ similar and $36 \%$ identical to the Cf9 C. fulvum disease resistance gene of tomato (Jones et al. 1994). No other cDNA clones with homology to known disease resistance genes were identified.

The longest of the three cDNA clones, 8-1, was used as probe on a Southern blot of EcoRI-digested BAC clones of the whole resistant and susceptible BAC contigs (including the clones not cosegregating with $V f$ resistance). The probe hybridized to multiple bands on all but two clones of the contig, comprising the clones not cosegregating with resistance on the resistant and the susceptible chromosome. The high number of hybridizing bands indicated that a gene cluster of many $C f$ resistance gene homologs is present in the $V f$ region. We called the members of the cluster "HcrVf genes" (homologs to $C$. fulvum resistance genes of the $V f$ region), in analogy to Parniske et al. (1999). This meant that the three $C f$ homolog cDNA clones (8-1, 10-5, and 15-4) could be derived from any of the BAC clones of the $V f$ region (from either the resistant or susceptible chromosome) or from genomic regions other than the $V f$ locus, in analogy to the presence of various $C f$ gene clusters in tomato (Parniske et al. 1997) and because apple is a species of ancient allotetraploid origin, which was confirmed by the finding that most restriction fragment length polymorphism markers hybridize to two loci located on two different chromosomes (Maliepaard et al. 1998).

\section{Sequence assembly and genetic mapping of $H c r V f$ genes.}

To concentrate our effort in finding candidate $V f$ genes exclusively on $C f$ homologs from the resistant chromosome at the $V f$ locus, we constructed Sau3AI and EcoRI subclone libraries of the five BAC clones used for the cDNA library screening and probed them with cDNA 8-1. After partially 
sequencing the subclones hybridizing to cDNA 8-1, we realized that many sequences were so similar to each other that it was impossible to assign them to different open reading frames (ORFs). Only two EcoRI subclones of BAC clone M18-2 could be assigned to one single ORF because these two subclones corresponded in length to the two EcoRI fragments of M18-1 (BAC clone overlapping with M18-2) that had hybridized to cDNA 8-1 in the Southern blot experiment described above. This ORF was called HcrVfl. We were able to align the other subclone sequences with HcrVfl, but as a result of the high homology between sequences, it was still impossible to assign the subclones to separate genes. We observed, however, that homology between various subclone sequences continued upstream of the putative start codon and downstream of the putative stop codon of HcrVfl. We used primers designed on these conserved sequences upstream and downstream of $\mathrm{HcrVfl}$ and were able to amplify two complete ORFs (named HcrVf2 and HcrVf3) in addition to HcrVfl. Figure 1B shows the region of the resistant contig from which the HcrVf genes were amplified. Because HcrVfl and HcrVf2 are located on BAC clone M18-2, which has one end separated from the $V f$ locus by two recombinants in our mapping populations, it was necessary to determine whether HcrVfl and HcrVf2 cosegregate with $V f$ resistance. We determined that HcrVf2 cosegregates with $V f$ resistance but were unable to find primers polymorphic for HcrVfl in our crosses. It has thus not yet been determined whether HcrVfl cosegregates with $V f$ resistance.
To determine whether there are more HcrVf genes on the five BAC clones cosegregating with $V f$ resistance, we performed a Southern blot of EcoRI-DraI-digested BAC clones of the $V f$ region probed with a 118 -bp polymerase chain reaction (PCR) fragment amplified with primers designed on the most conserved region among the three HcrVf genes that is not digested by either EcoRI or DraI (data not shown). Five different restriction fragments of the five BAC clones spanning the $V f$ region hybridized to this probe (Fig. 2). Thus, this is the minimum number of $H c r V f$ genes on these five BAC clones, although because some HcrVf genes may have EcoRIDraI fragments of the same size, this number may be higher.

\section{Transcription analysis of $\mathrm{HcrVf1}, \mathrm{HcrVf2}$, and $\mathrm{HcrVf3}$.}

Because the sequences of cDNAs 8-1, 10-5, and 15-4 did not correspond to $H c r V f 1, H c r V f 2$, or $H c r V f 3$, we used reverse transcription (RT)-PCR to determine their transcription. We used $H c r V f l$-specific primers, bulked the RNA extracted from leaves harvested at different time points after inoculation with $V$. inaequalis, and obtained three RT-PCR bands from the RNA of Prima and Florina, two scab-resistant cultivars that are heterozygous at the $V f$ locus. One of the three bands was missing when the scab-susceptible cultivar Golden Delicious was used as template. Cloning and sequencing of this product from Prima and Florina showed a 100\% match with each other as well as with HcrVfl, which is therefore transcribed. We used $H c r V f$ - and $H c r V f 3$-specific primers and obtained single

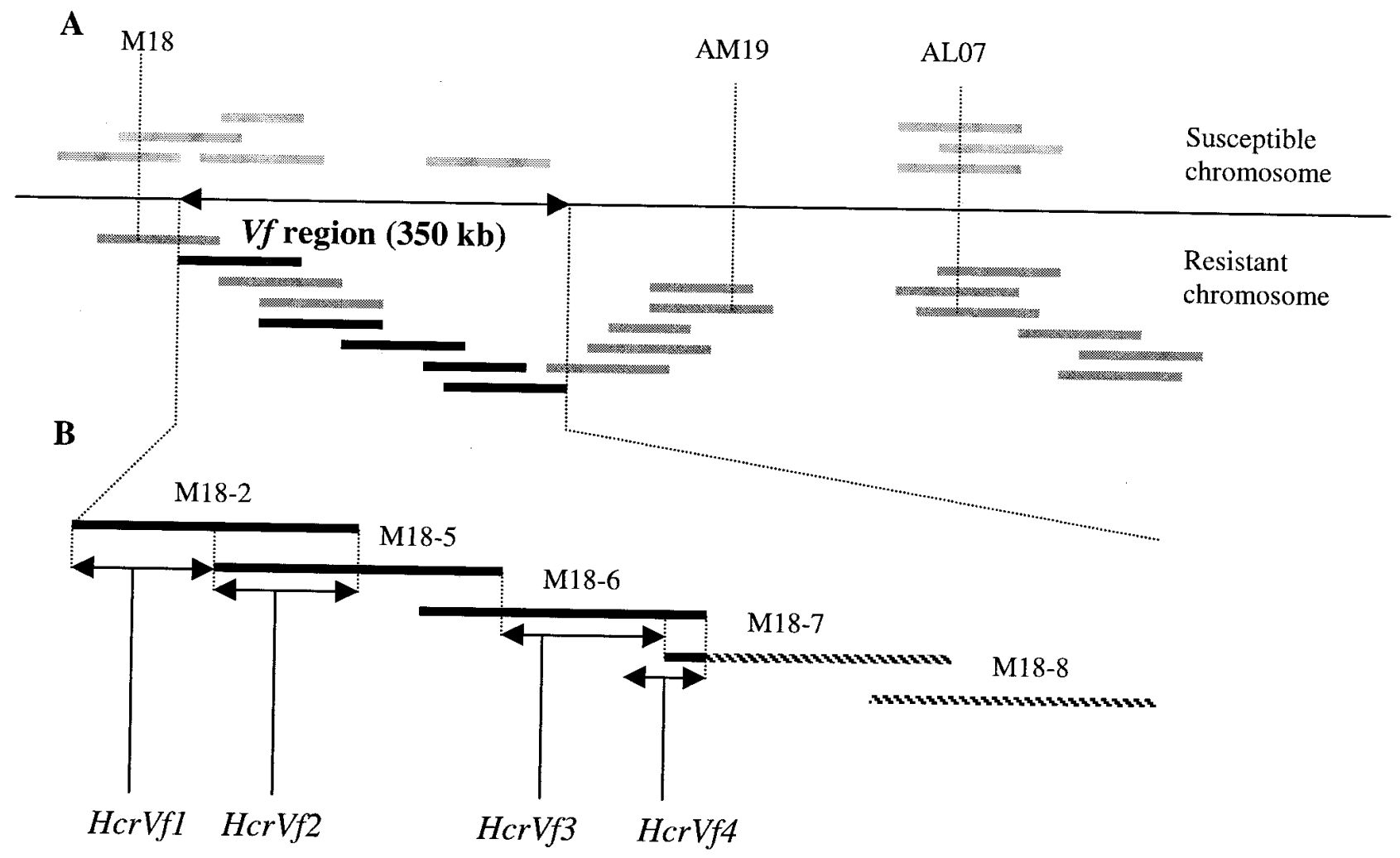

Fig. 1. A, Schematic representation of the $V f$ region. Dark-gray bars indicate the bacterial artificial chromosome (BAC) clones derived from the chromosome carrying the $V f$ allele at the $V f$ locus ("resistant" chromosome), whereas light-gray bars indicate the BAC clones derived from the homologous chromosome ("susceptible" chromosome). The $V f$ locus was mapped to a $350 \mathrm{~kb}$ region covered by a minimum of five BAC clones, indicated in black (Patocchi et al. 1999b). Markers M18, AM19, and AL07 were the starting points of the chromosome walk that led to the construction of the BAC contigs. BAC clones are not drawn to scale. B, Relative positions (on the resistant contig) of the four characterized HcrVf genes (homologs of Cladosporium fulvum resistance genes of the $V f$ region). Hatched horizontal bars represent the part of the $V f$ region that does not contain $H c r V f$ genes. 
bands in the two $V f$ cultivars that were missing in the susceptible Golden Delicious. The sequences of the HcrVf2 RT-PCR products matched the genomic HcrVf2 sequence and confirmed transcription of $H c r V f 2$. The $H c r V f 3$ RT-PCR products showed a $97 \%$ match with HcrVf3. Alignment with the sequenced BAC subclones, however, showed a $100 \%$ match with a 5,000-bp EcoRI subclone of M18-6. Therefore, transcription of this additional gene, HcrVf4, was shown. HcrVf3 may be a pseudogene or have a very low transcription level compared with HcrVf4. Figure 1B shows the approximate location of HcrVf4 in the BAC contig.

We then performed RT-PCR experiments on the RNA extracted from uninfected leaves of Florina and Prima and determined that HcrVfl, HcrVf2, and HcrVf4 also are transcribed in the absence of a pathogen attack.

\section{Determination of transcription and translation start of the HcrVf genes.}

We used 5' rapid amplification of cDNA ends (RACE) on $H c r V f l$ and $H c r V f 2$ to verify the predicted translation start for the ORFs of the HcrVf genes. By cloning and sequencing several $5^{\prime}$ RACE products obtained with primers designed in regions common to HcrVfl and HcrVf2, we identified two clones showing a perfect match with the genomic sequence of $H c r V f 2$, comprising the first 244 bases of the ORF and the 36 bases upstream of the putative start codon, indicating that the leader sequence of $H c r V f 2$ might be 36 bases. A putative TATA box 26 bases from the presumed transcription start and a CAAT box 22 bases upstream of the TATA box were identified. Upstream of the presumed transcription start, a 26-base, CT-rich sequence was found with no correspondence to the upstream sequence of HcrVf2. According to Roche (Rotkreuz, Switzerland), CT-rich sequences can be RACE-PCR artifacts as a result of the slippage of the poly(T) primer during amplification. From our data, it is not possible to determine whether this hypothesis is correct. The presence of a 280-bp perfect match between the RACE clones and the genomic sequence of $H c r V f 2$, however, strongly supports the idea that the sequence of the two RACE clones is the $5^{\prime}$-end of the HcrVf2 transcript and, as a result of the high homology between the members of the HcrVf cluster, the ORFs that we predict for HcrVfl and HcrVf3 also should be correct.

\section{HcrVf1 through $H c r V f 3$ are predicted to be membrane-bound glycoproteins with an extracellular leucine-rich repeat domain.}

The deduced amino acid sequences of HcrVfl, HcrVf2, and HcrVf3 are 1,015, 980, and 916 amino acids, respectively (Fig. 3). Although the DNA sequences of the sequenced ORFs of the HcrVf genes have, on average, only $44 \%$ identity to the tomato $C f 9 C$. fulvum disease resistance, the proteins predicted for the HcrVf genes show the same domains as those predicted for the $C f 9$ resistance gene (Jones et al. 1994). A signal peptide and its cleavage site is predicted to be present at the $\mathrm{N}$ terminus of the three proteins (domain $\mathrm{A}$ ). Domain $\mathrm{B}$ is thus the $\mathrm{N}$ terminus of the mature protein, once the signal peptide has been cleaved. The $\mathrm{C}$ domains of the HcrVf genes 1 through 3 contain 30, 29, and 26 imperfect LRRs, respectively. The average length of the LRRs is 24 amino acids, and each LRR contains the consensus sequence LxxLxxLxxLx LSxNxLxGxIP. The glycine in the consensus sequence indi- cates that the LRRs are of extracellular nature (Jones et al. 1994). As for the $C f$ proteins, no function could be assigned to domain D, which is composed of 27 amino acids. Domain F, composed of 36 hydrophobic amino acids, is predicted to be the transmembrane domain of the protein. Domains E and G, flanking the hydrophobic domain, are rich in polar amino acids, which is consistent with their predicted role of orienting and anchoring the protein to the cell membrane. Many putative $\mathrm{N}$-glycosylation sites are present in domains $\mathrm{B}$ to $\mathrm{D}$ of HcrVf1 (26 sites), HcrVf2 (23 sites), and HcrVf3 (23 sites), respectively. Therefore, similar to the $C f$ resistance genes, the $H c r V f$ genes probably encode extracytoplasmic glycoproteins anchored to the cell membrane, with the majority of the extracytoplasmic domain consisting of LRR motifs.

At the amino acid level, the three complete ORFs compared two by two show, on average, $87 \%$ identity. The most conserved region among the three ORFs is domain $\mathrm{A}$ and the region spanning from domain $\mathrm{D}$ to $\mathrm{G}$, presenting, on average, 95 and $96 \%$ identity, respectively. The more variable domains are $\mathrm{B}$ and $\mathrm{C}$, presenting, on average, 84 and $86 \%$ identity, respectively. Furthermore, the variability is not distributed equally within domain $\mathrm{C}$, which can be divided into subdomain C2 (89\% identity between the three considered $\mathrm{HcrVf}$

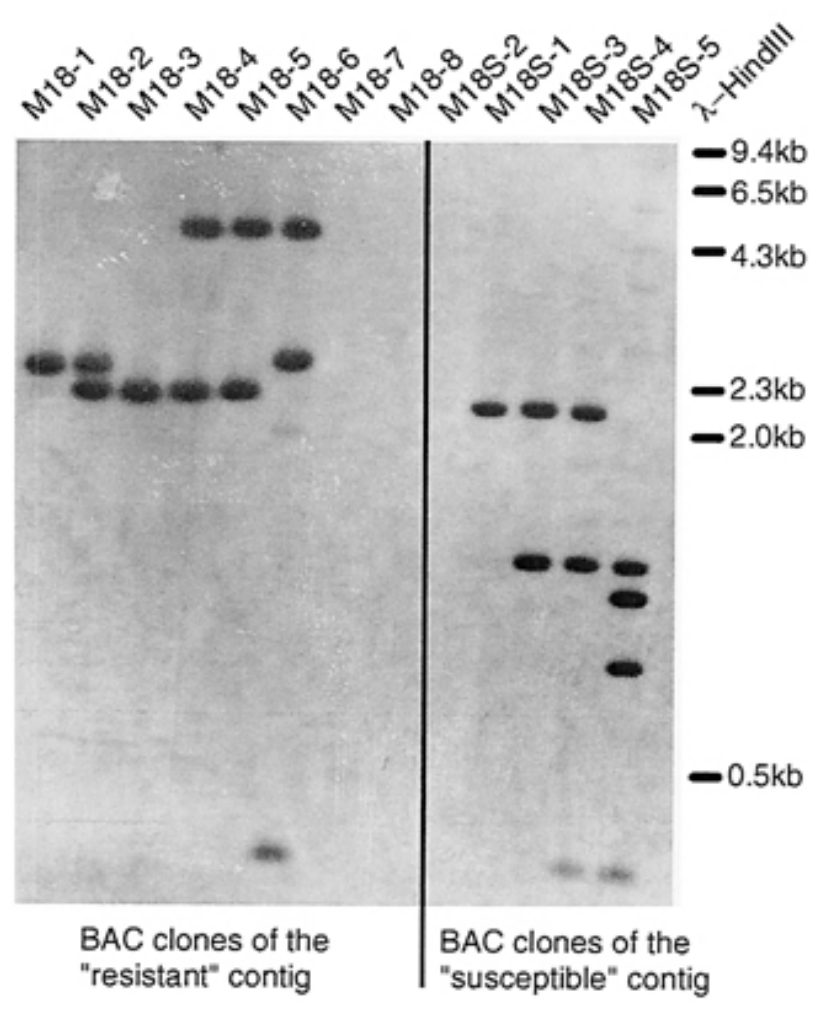

Fig. 2. At least five HcrVf genes are located on the bacterial artificial chromosome (BAC) clones cosegregating with $V f$ resistance. A 118-bp probe derived from the highly conserved domains $\mathrm{E}$ and $\mathrm{F}$ of HcrVf2 was hybridized to EcoRI-DraI double-digested BAC clones spanning the Vf region. The probe hybridizes to five bands of the $\mathrm{BAC}$ clones from the "resistant" chromosome cosegregating with Vf resistance and to an additional five of the corresponding BAC clones from the "susceptible" chromosome. Each of the bands corresponds to at least one HcrVf gene (because some HcrVf genes may have EcoRI-DraI fragments of the same size, this number may be higher). Other HcrVf sequences were found on BAC clones AM19-1 through AM19-4 and AL07-1 through AL07-3 (not cosegregating with $V f$ resistance; data not shown). 
genes) and $\mathrm{C} 1$ (82\% identity between the three considered HcrVf genes). The lower level of identity in $\mathrm{C} 1$ is not only a result of the difference in the number of LRRs (with reference to HcrVf1, HcrVf2 lacks two and HcrVf3 lacks five LRRs, respectively), but also to the higher number of amino acid differences observed within a single LRR.

Hor genes are present in multiple loci in the apple genome.

Comparing the HcrVf genomic sequences with the BAC end sequences isolated during the construction of the $V f$ BAC contig (Patocchi et al. 1999b), we noticed that the AL07-2Sp6 BAC end probe, which had hybridized to most BAC clones of the $V f$ contig, including the BAC clones of the distinct AL07 contig separated from the $V f$ locus by $1.2 \mathrm{cM}$ (Patocchi et al. 1999b), showed 93\% identity with the HcrVf genes. This probe was used to screen the apple BAC library, and 11 clones were identified that did not map to the $V f$ region: 15J14, 40O10, 43F22, 49J15, 55I7, 57G7, 62H3, 72M18, 76G16, 90N4, 94H24. This result shows that $\mathrm{Hcr}$ sequences also are present in other loci of the apple genome.

\section{DISCUSSION}

\section{Identification of candidate $V f$ genes with homology} to the tomato $\boldsymbol{C f}$ resistance gene family.

We identified a cluster of $C f$ homologs at the $V f$ locus. ORFs of three members of the cluster, HcrVf1, HcrVf2, and HcrVf3, which were completely sequenced, a fourth ORF
(HcrVf4) that has been partially sequenced, and the presence of at least one more HcrVf gene, which can be deduced from the Southern blot experiment (Fig. 2). HcrVf1, HcrVf2, and $H c r V f 4$ are transcribed genes, and HcrVf3 is probably a nonexpressed pseudogene. HcrVf2, HcrVf3, and HcrVf4 cosegregate with $V f$ resistance in our mapping populations (2,071 plants), whereas the map position of HcrVfl has yet to be determined. Because HcrVf3 is a pseudogene, to date only $H c r V f 2$ and $H c r V f 4$ are candidate $V f$ genes.

The comparison of the deduced amino acid sequences of HcrVfl through HcrVf3 with the deduced amino acid sequence of the tomato $H c r 9$ genes clearly shows the same type of overall protein structure (Fig. 3). It is possible to divide the predicted apple HcrVf proteins and the tomato Hcr 9 proteins in the same domains. Additionally, the level of conservation and variability of the different domains between the members of each of the two families is similar. Domains A, D, E, F, and $\mathrm{G}$ are very conserved, whereas domains $\mathrm{B}$ and $\mathrm{C}$ are more variable. In both gene families, domain $\mathrm{C}$ can be divided into two subdomains, $\mathrm{C} 1$ and $\mathrm{C} 2$, on the basis of degrees of conservation, with $\mathrm{C} 1$ being more variable than $\mathrm{C} 2$. Parniske et al. (1997) propose that the region of the Hcr9 genes corresponding to the sixteen LRRs closest to the $\mathrm{N}$ terminus (domain $\mathrm{C} 1$ in the HcrVf gene family) encodes the region of the proteins involved in the recognition of the avirulence product of the pathogen. For this reason, the region is evolving more rapidly and is, therefore, less conserved, probably to adapt to the evolution of the pathogen.

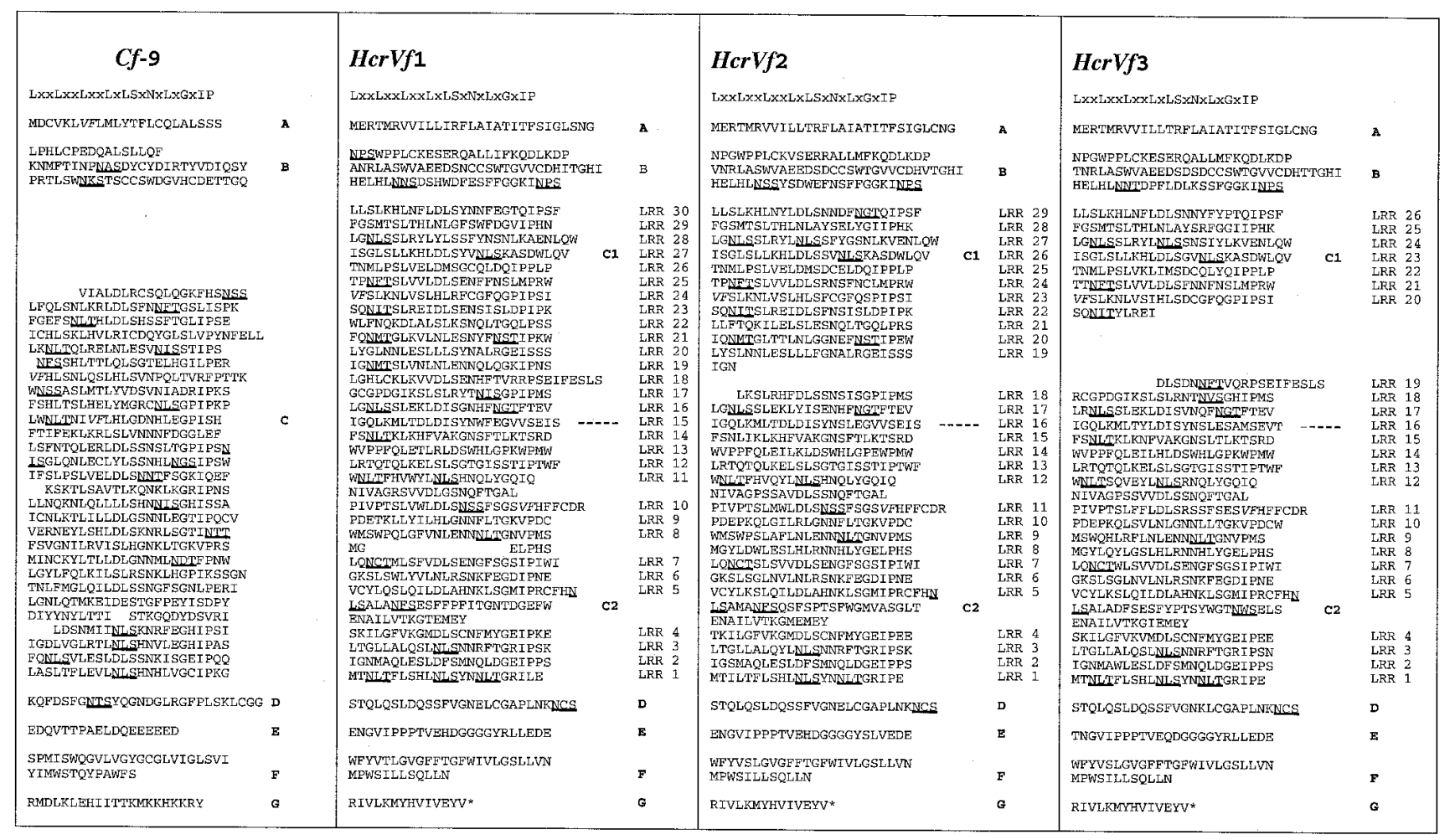

Fig. 3. Comparison of the predicted amino acid sequences of $C f 9, H c r V f 1, H c r V f 2$, and $H c r V f 3$ (HcrVf4 is not shown because its sequence is incomplete). The $C f-9$ resistance gene was divided into seven domains. Domain A is predicted to be a signal peptide, $\mathrm{B}$ is the presumed $\mathrm{NH}_{2}$ terminus of the mature protein, $\mathrm{C}$ is a leucine-rich repeat (LRR) domain, $\mathrm{D}$ is of unknown function, $\mathrm{E}$ is an acidic domain, $\mathrm{F}$ is hydrophobic and predicted to be a transmembrane domain, and $\mathrm{G}$ is a basic domain representing the $\mathrm{C}$ terminus (Jones et al. 1994). The same seven domains also are predicted for the three $H c r V f$ genes. $\mathrm{C} 1$ and $\mathrm{C} 2$ are the two subdomains of domain $\mathrm{C}$. The putative $\mathrm{N}$-glycosylation sites are underlined. 
Is one member of the Hcr $\boldsymbol{H} \boldsymbol{f}$ gene cluster the $\boldsymbol{V} \boldsymbol{f}$ gene?

After sequencing 50 different cDNAs hybridizing to the five BAC clones of the $V f$ locus, we cannot exclude that there are other genes in the region. An indication that the 750,000 cDNAs screened may not have been sufficient to detect all the genes of the region is the fact that we did not detect the cDNAs of HcrVf1, 2, and 4, which are transcribed genes. The way to determine the total number of genes present at the $V f$ locus will be to completely sequence the five BAC clones of the region. Therefore, we cannot exclude that there are candidate $V f$ genes besides the HcrVf genes in the $V f$ region. Anyway, in addition to the homology of the HcrVf genes at the $V f$ locus of apple with the tomato Hcr 9 genes, there are other similarities between $V f$ apple scab resistance and $C f$ tomato $C$. fulvum resistance that cause us to believe that one of the members of the HcrVf gene cluster may be the determinant of $V f$ resistance. On the host side, $V f$ apple scab resistance is influenced by the genetic background and has different resistance phenotypes, ranging from no symptoms to restricted sporulating lesions (Chevalier et al. 1991; Gessler et al. 1989). Also, the $C f$ resistance phenotype depends on the genetic background (Hammond-Kosak et al. 1994). Scab resistance is found on older leaves of all apple varieties (ontogenic resistance) (Gessler and Stumm 1984). Parniske et al. (1997) reported that certain $\mathrm{Hcr} 9$ genes in tomato give resistance in older leaves but not in younger leaves. By analogy, we can hypothesize that ontogenic resistance of apple involves the $H c r V f$ homologs present at the $V f$ locus and at other loci of all apple cultivars. On the pathogen side, $C$. fulvum and $V$. inaequalis are extracellular pathogens, which makes it plausible that an extracellular LRR recognition domain is involved in $V f$ resistance as it is in $C f$ resistance. It also may be possible that more than one member of the HcrVf gene cluster at the $V f$ locus has LRR domains that recognize the same (or different) avirulence protein(s) of $V$. inaequalis.

Because the HcrVf alleles from the resistant chromosome at the $V f$ locus are derived from the wild apple species $M$. floribunda and those from the susceptible chromosome are derived from Malus $\times$ domestica cultivars such as Golden Delicious, the two homologous chromosomes at the $V f$ locus are highly polymorphic. Thus, it is not possible to identify a single mutation in one of the HcrVf alleles on the resistant chromosome compared with the corresponding HcrVf allele on the susceptible chromosome to determine which HcrVf gene is the Vf gene. Therefore, the absence of RT-PCR products for HcrVfl, $H c r V f 2$, or HcrVf3 in Golden Delicious cannot be interpreted as an indication that one of these genes determines $V f$ resistance. The ultimate answer to the identity of the $V f$ gene will be obtained only from the transformation, now in progress, of susceptible apple cultivars with the single HcrVf genes that are transcribed and cosegregates with $V f$ resistance. Moreover, if we obtain scab-resistant transformants from high-quality commercial cultivars, we will have identified the $V f$ gene and, at the same time, created scab-resistant, high-quality apple cultivars.

\section{Can $\mathrm{Hcr} 9$ homologs contribute to the creation of durable scab resistance in apple?}

A $V f$ scab-resistant cultivar has a limited value because $V f$ scab resistance has already been overcome in some regions of Europe (Bénaouf and Parisi 2000; Parisi et al. 1993). There- fore, it is necessary to combine other scab resistance genes with $V f$ resistance. We have shown that the apple genome contains several Hcr genes in the $V f$ region (on the resistant and susceptible chromosomes) as well as in other regions that are different from the $V f$ locus. These $\mathrm{Hcr}$ genes could represent the ephemeral resistance genes present in all apple cultivars that are effective against monoclonal isolates of $V$. inaequalis and/or other broad-range apple scab resistance genes from wild relatives of the cultivated apple (Williams and Kuc 1969). Some of these genes have been mapped with molecular markers (Cheng et al. 1998; Gardiner et al. 1999). The sequence information of the HcrVf genes can now be used in an approach similar to the candidate gene approach used with the NBS-LRR genes in other plant species (Aarts et al. 1998; Kanazin et al. 1996; Leister et al. 1996; Meyers et al. 1998; Yu et al. 1996) to map and identify candidate genes for apple scab resistance genes. This could accelerate scab resistance gene cloning and, consequently, the creation of durable disease-resistant apple varieties as a result of scab resistance gene pyramiding.

\section{MATERIALS AND METHODS}

\section{Plant material and plant inoculation.}

Plants of the scab-resistant Florina (heterozygous for the $V f$ gene) budded on M9 root stock were inoculated with a conidial suspension of $V$. inaequalis, as described by Gianfranceschi et al. (1996). The inoculum was collected from susceptible seedlings of different crosses at Wadenswil (FAW, Swiss Federal Research Station). The first three leaves on each shoot were collected 24 or $48 \mathrm{~h}$ after incubation at $18^{\circ} \mathrm{C}$ and $100 \%$ humidity. They were dipped immediately in liquid nitrogen and stored at $-80^{\circ} \mathrm{C}$ until use. Noninoculated leaves also were collected and stored in the same way.

\section{cDNA library construction and screening.}

mRNA was extracted from $0.5 \mathrm{~g}$ of apple leaves with the Poly(A)Pure mRNA isolation kit (Ambion, Austin, TX, U.S.A.), following the manufacturer's instructions. mRNA quality was tested by gel electrophoresis in accordance with Sambrook et al. (1989). cDNA was synthesized with the cDNA Synthesis Kit (Stratagene, La Jolla, CA, U.S.A.). The kit's instructions were followed, except rather than using the kit's columns, cDNA was size selected on a $1 \%$ agarose mini gel in $1 \times$ TAE. After a 30 -min run at $5 \mathrm{~V}$ per cm, the marker lane was stained with ethidium bromide and the cDNA between the loading slot and the 500-bp marker band was cut from the gel. The cDNA was purified with the GENECLEAN kit (Bio101, Joshua Way, CA, U.S.A.). cDNA was then cloned and packaged, following the manufacturer's instructions.

For library screening, phages were plated at a density of 20,000 PFU per 150-mm plate. Plaques were transferred to Circular Hybond $\mathrm{N}+$ nylon filters, following standard procedures (Sambrook et al. 1989).

To probe the cDNA libraries with the BAC clones spanning the $V f$ region, BAC DNA inserts were isolated by digesting the BAC DNAs with EcoRI (the enzyme used for cloning). The restriction fragments were run on an $0.8 \%$ agarose gel, the vector band was cut from the gel, the voltage was inverted, and the insert fragments were cut from the gel, once they had again nearly reached the loading slot. The fragments were 
extracted from the gel with the GENECLEAN kit, and $200 \mathrm{ng}$ of BAC DNA inserts were labeled with ${ }^{32} \mathrm{P}$ dCTP in a $200-\mu \mathrm{l}$ vol with the random priming labeling kit (GIBCO BRL, Rockville, MD, U.S.A.). The probe was added to the plaque filters that had been prehybridized for $24 \mathrm{~h}$ at $68^{\circ} \mathrm{C}$ in hybridization solution $(5 \times \mathrm{SSC}[1 \times \mathrm{SSC}$ is $0.15 \mathrm{M} \mathrm{NaCl}$ plus 0.015 M sodium citrate]; $0.5 \%$ SDS [sodium dodecyl sulfate]; 25 $\mathrm{mM}$ potassium phosphate buffer, $\mathrm{pH} 6.5$; and $5 \times$ Denhardt's). Hybridization was then carried out for $24 \mathrm{~h}$ at $68^{\circ} \mathrm{C}$. Filters were washed once at $68^{\circ} \mathrm{C}$ for $15 \mathrm{~min}$ in $2 \times \mathrm{SSC}, 0.1 \% \mathrm{SDS}$, and twice for $30 \mathrm{~min}$ at $68^{\circ} \mathrm{C}$ in $0.5 \times \mathrm{SSC}, 0.1 \% \mathrm{SDS}$. Filters were exposed for 3 days to $\mathrm{X}$-ray films at $-80^{\circ} \mathrm{C}$. Plaque regions corresponding to signals detected on the X-ray film were subjected to secondary screening after separate in vivo excisions for each of the plaque regions, and 200 colonies from each plaque region were used for the secondary screen. Positive colonies from the secondary screen were confirmed by an additional hybridization. DNA extracted from positive clones was sequenced with M13 primers with the BigDye kit (PE Applied Biosystems, Foster City, CA, U.S.A.) and run on an ABI 373 DNA sequencing system (PE Applied Biosystems). Sequences were analyzed with GCG software (Genetics Computer Group, Madison, WI, U.S.A.) and compared with GenBank by BLASTX and Fasta (Altschul et al. 1997).

\section{BAC subcloning and screening.}

BAC DNA was digested with either Sau3AI or EcoRI for 3 $\mathrm{h}$, and Sau3AI digests were cloned directly in pGEM-4z vector (Promega, Madison, WI, U.S.A.). EcoRI digests were size selected on an $0.8 \%$ agarose gel in order to increase the representation of clones with large inserts, and three different slices (fragments longer than 4,000 bp, between 4,000 and $500 \mathrm{bp}$, and shorter than $500 \mathrm{bp}$ ) were gel purified with the Qiagen gel purification kit (Basel, Switzerland) and ligated separately to the pGEM-4z vector. Ligations were electroporated into Escherichia coli DH10 cells (GIBCO BRL), Sau3AI and EcoRI subclones were transferred to 384-well plates, and highdensity colony filters were prepared with a Beckmann 2000 (Fullerton, CA, U.S.A.) automated workstation, in accordance with Zhang et al. (1996). Prehybridization, hybridization, and washes of subclone filters were performed as described for the phage filters. A gel-purified PCR product amplified from cDNA clone 8-1 was used as probe. Positive subclones were sequenced, and sequences were analyzed as described for the cDNA clones. Primers developed on subclone sequences were designed with the Primer 3.0 program (Whitehead Institute of Biomedical Research, Cambridge, MA, U.S.A.) and synthesized from Microsynth (Balglach, Switzerland). Primers HcrVf1-for (GCGGTCTCATGCCGTAAAG) and HcrVf1-rev (TACTGGCATATTCGTCGCAG) were used to amplify the complete genomic sequence of HcrVfl, HcrVf2, and HcrVf3 from the BAC clones spanning the $V f$ genomic region. The annealing temperature of both primers was $60^{\circ} \mathrm{C}$.

\section{Estimation of the number of $\boldsymbol{H c r} \boldsymbol{V} \boldsymbol{f}$ s at the $\boldsymbol{V} \boldsymbol{f}$-region.}

Southern blots carrying EcoRI and DraI double-digested BAC DNA, extracted in accordance with Patocchi et al. (1999b), were hybridized with a probe developed in the highly conserved domains $\mathrm{E}$ and $\mathrm{F}$ of $\operatorname{HcrVfl}$, 2, and 3. The probe was amplified from $H c r V f 2$ with the primers domainE-for
(GGTTCTACGTGAGCTTGGGA) and domainF-rev (AGC ACTATCCTATTCAGCAACTG).

\section{RT-PCR.}

Leaves from cultivars Florina (Vfvf), Prima (Vfvf), and Golden Delicious ( $v f v f$ ) were inoculated with $V$. inaequalis, as described by Gianfranceschi et al. (1996), and harvested 0, 12, 24, 48, and $96 \mathrm{~h}$ postinoculation. Total RNA was extracted from approximately $25 \mathrm{mg}$ of leaf tissue with the Promega SV Total RNA Isolation System, following the manufacturer's instructions. The Qiagen OneStep RT-PCR kit was used to perform RT-PCR, following the manufacturer's instructions. The templates for RT-PCR (three bulks, one for each cultivar) were set up by mixing the same volumes of 1:50 dilutions of extracted total RNA of leaves harvested at different times, without previous quantification. To check for DNA contamination, control reactions were performed by inhibiting reverse transcriptase $\left(15 \mathrm{~min}\right.$ at $\left.95^{\circ} \mathrm{C}\right)$. Primers RT1-for (CAATGCCT TACGTGGTGAAA) and domainF-rev (AGCACTATCTAT TCAGCAAYYSTG) were used to amplify HcrVfl RT-PCR products. Primers RT1-for and RT2-rev (CAGGGATTCCA GCCAATCTA) were used for the amplification of HcrVf2 RTPCR products, whereas primers RT3-for (TCACGGTTCAA AGACCATCC) and RT3-rev (GCAGGGATCCCAGATAT TGC) were used for HcrVf3. The annealing temperature of all primers was $60^{\circ} \mathrm{C}$. The primers used for transcription analysis of HcrVf2 also were used for its genetic mapping.

Single RT-PCR products were purified with the Qiagen QIAquick PCR purification kit and sequenced. Sequencing and sequence analysis were performed as described above. When RT-PCR reactions produced several bands, the bands of the expected size were gel purified and cloned into pGEM-T vector with the Promega pGEM-T vector system.

\section{RACE.}

RACE experiments were performed with the Roche $5^{\prime}-3^{\prime}$ RACE kit in combination with the Roche Expand High Fidelity PCR System, following the manufacturer's instructions. Florina RNA bulk, prepared for the RT-PCR experiments, was used as template. The three specific RACE primers, SP1 HcrVf (CAACCAGTCAGATGCTTTG), SP2 HcrVf (TGC TTTAAACTGAGCAAAGAAGG), and SP3 HcrVf (TAT TAAGGTGCAGCTCGTGG), necessary for the amplification of the $5^{\prime}$ gene ends, were designed in regions common to the genomic sequences of HcrVfl and HcrVf2. RACE products were run on a $1 \% 0.5 \times$ Tris-borate-EDTA agarose gel, and bands of the expected size were gel purified and blunt-end cloned with the Roche PCR cloning kit.

\section{ORF analysis and protein prediction.}

ORFS were predicted with ORF Finder. Putative signal peptide cleavage sites and putative transmembrane domains of the HcrVf genes were predicted with the CBS prediction server. $\mathrm{N}$-glycosylation sites were predicted in accordance with Alberts et al. (1990).

\section{ACKNOWLEDGMENTS}

The authors are grateful for the financial support made available by the Laimburg Center for Agricultural and Forestry Experimentation, Autonomous Province of Bolzano-Bozen, Italy, and the Swiss Federal Institute of Technology, grant 0-20-354-96. 


\section{LITERATURE CITED}

Aarts, M. G. M., Hekkert, B. t. L., Holub, E. B., Beynon, J. L., Stiekema, W. J., and Pereira, A. 1998. Identification of R-gene homologous DNA fragments genetically linked to disease resistance loci in Arabidopsis thaliana. Mol. Plant-Microbe Interact. 11:251258.

Alberts, B., Bray, D., Lewis, J., Raff, M., Roberts, K., and Watson, J. D. 1990. Molekularbiologie der Zelle, VHC Verlagsgesellschaft GmbH, Weinheim, Germany.

Altschul, S. F., Madden, T. L., Schaffer, A. A., Zhang, J., Zhang, Z., Miller, W., and Lipman, D. J. 1997. Gapped BLAST and PSI-BLAST: A new generation of protein database search programs. Nucleic Acids Res. 25:3389-3402.

Bénaouf, G., and Parisi, L. 2000. Genetics of host-pathogen relationships between Venturia inaequalis races 6 and 7 and Malus species. Phytopathology 90:236-242.

Boone, D. M. 1971. Genetics of Venturia inaequalis. Ann. Rev. Phytopathol. 9:297-318.

Cheng, F. S., Weeden, N. F., Brown, S. K., Aldwinckle, H. S. Gardiner, S. E., and Bus, V. G. 1998. Development of a DNA marker for $V m$, a gene conferring resistance to apple scab. Genome 41:208-214.

Chevalier, M., Lespinasse, Y., and Renaudin, S. 1991. A microscopic study of different classes of symptoms coded by the $V f$ gene in apple for resistance to scab (Venturia ineaqualis). Plant Pathol. 40:249-256.

Crandall, C. S. 1926. Apple breeding at the University of Illinois. Ill. Agric. Exp. Stn. Bull. 275:341-600.

Dayton, D. F., Mowry, J. B., Hought, L. F., Bailey, C. H., Williams, E. B., Janick, J., and Emerson, F. H. 1970. Prima: An early fall red apple with resistance to apple scab. Fruit Var. Hortic. Dig. 24:20-22.

Dixon, M. S., Jones, D. A., Keddie, J. S., Thomas, C. M., Harrison, K., and Jones, J. D. G. 1996. The tomato $C f$-2 disease resistance locus comprises two functional genes encoding leucine-rich repeat proteins. Cell 84:451-459.

Gardiner, S. E., Bus, V., Bassett, H., Goodman, M., Greer, L., Ranatunga, C., Rikkerink, E., and Forster, R. 1999. Identification of molecular markers linked to major resistances to apple scab, powdery mildew, and woolly apple aphid in apple. Proceedings of the VII Plant and Animal Genome Conference, San Diego.

Gessler, C. 1989. Genetics of the interaction Venturia inaequalis-Malus: The conflict between theory and reality. Pages 168-190 in: Integrated control of pome fruit diseases II. OILB-WPRS Bull. 6:12.

Gessler, C., and Stumm, D. 1984. Infection and stroma formation by Venturia inaequalis on apple leaves with different degrees of susceptibility to scab. Phytopathol. Z. 110:119-126.

Gianfranceschi, L., Koller, B., Seglias, N., Kellerhals, M., and Gessler, C. 1996. Molecular selection in apple for resistance to scab caused by Venturia inaequalis. Theor. Appl. Genet. 93:199-204.

Hammond-Kosack, K. E., Jones, D. A., and Jones, J. D. G. 1994. Identification of two genes required in tomato for full $C f$-9-dependent resistance to Cladosporium fulvum. Plant Cell 6:361-374.

Jones, D. A., Thomas, C. M., Hammond-Kosack, K. E., Balint-Kurti, P. J., and Jones, J. D. G. 1994. Isolation of the tomato $C f-9$ gene for resistance to Cladosporium fulvum by transposon tagging. Science 266:789-793.

Kanazin, V., Marek, L. F., and Shoemaker, R. C. 1996. Resistance gene analogs are conserved and clustered in soybean. Proc. Natl. Acad. Sci. USA 93:11746-11750.

Koch, T., Kellerhals, M., and Gessler, C. 2000. Virulence pattern of Venturia inaequalis field isolates and corresponding differential resistance in Malus $\times$ domestica. J. Phytopathol. 148:357-364.

Laurens, F. 1996. Review of the current apple breeding programs in the world: Objectives for scion/cultivars improvement. Acta Hortic. 484:163-170.
Leister, D., Ballvora, A., Salamini, F., and Gebhardt, C. 1996. A PCRbased approach for isolating pathogen resistance genes from potato with potential for wide application in plants. Nature Genet. 14:421-429.

Maliepaard, C., Alston, F. H., Van Arkel, G., Brown, L. M., Chevreau, E., Dunemann, F., Evans, K. M., Gardiner, S., Guilford, P., Van Heusden, A. W., Janse, J., Laurens, F., Lynn, J. R., Manganaris, A. G., Den Nijs, A. P. M., Periam, N., Rikkerink, E., Roche, P., Ryder, C., Sansavini, S., Schmidt, H., Tartarini, S., Verhaegh, J. J., Vrielink-Van Ginkel, M., and King, G. J. 1998. Aligning male and female linkage maps of apple (Malus pumila Mill.) using multi-allelic markers. Theor. Appl. Genet. 97:60-73.

Meyers, B. C., Chin, D. B., Shen, K. A., Sivaramakrishnan, S., Lavelle, D. O., Zhang, Z., and Michelmore, R. W. 1998. The major resistance gene cluster in lettuce is highly duplicated and spans several megabases. Plant Cell 10:1817-1832.

Parisi, L., Lespinasse, Y., Guillaumes, J., and Krüger, J. 1993. A new race of Venturia inaequalis virulent to apples with resistance due to the $V f$ gene. Phytopathology 83:533-537.

Parniske, M., Hammond-Kosack, K. E., Golstein, C., Thomas, C. M., Jones, D. A., Harrison, K., Wulff, B. B. H., and Jones, J. D. G. 1997. Novel disease resistance specificities result from sequence exchange between tandemly repeated genes at the $C f-4 / 9$ locus of tomato. Cell 91:821-832.

Parniske, M., Wulff, B. B. H., Bonnema, G., Thomas, C. M., Jones, D. A., and Jones, J. D. G. 1999. Homologues of the $C f-9$ disease resistance gene $(H c r 9 s)$ are present at multiple loci on the short arm of tomato chromosome 1. Mol. Plant-Microbe Interact. 12:93-102.

Patocchi, A., Gianfranceschi, L., and Gessler, C. 1999a. Towards the map-based cloning of $V f$ : Fine and physical mapping of the $V f$-region. Theor. Appl. Genet. 99:1012-1017.

Patocchi, A., Vinatzer, B. A., Gianfranceschi, L., Tartarini, S., Zhang, H. B., Sansavini, S., and Gessler, C. 1999b. Construction of a $550 \mathrm{~kb}$ BAC contig spanning the genomic region containing the apple resistance gene $V f$. Mol. Gen. Genet. 262:884-891.

Sambrook, J., Fritsch, E. F., and Maniatis, T. 1989. Molecular Cloning: A Laboratory Manual, 2nd ed. Cold Spring Harbor Laboratory, Cold Spring Harbor, NY, U.S.A.

Sierotzki, H., and Gessler, C. 1998. Inheritance of virulence of Venturia inaequalis toward Malus $\times$ domestica cultivars. J. Phytopathol. 146:509-514.

Sierotzki, H., Eggenschwiler, M., Boillat, O., McDermott, J. M., and Gessler, C. 1994. Detection of variation in virulence toward susceptible apple cultivars in natural populations of Venturia inaequalis. Phytopathology 84:1005-1009.

Staskavicz, B. J., Ausubel, F. M., Baker, B. J., Ellis, J. G., and Jones, J. D. G. 1995. Molecular genetics of plant disease resistance. Science 268:661-667.

Thomas, C. M., Jones, D. A., Parniske, M., Harrison, K., Balint Kurti, P. J., Hatzixanthis, K., and Jones, J. D. G. 1997. Characterization of the tomato $C f-4$ gene for resistance to Cladosporium fulvum identifies sequences that determine recognitional specificity in $C f-4$ and $C f-9$. Plant Cell 9:2209-2224.

Vinatzer, B. A., Zhang, H.-B., and Sansavini, S. 1998. Construction and characterization of a bacterial artificial chromosome library of apple. Theor. Appl. Genet. 97:1183-1190.

Williams. E. B., and Kuc. J. 1969. Resistance in Malus to Venturia inaequalis. Ann. Rev. Phytopathol. 7:223-246.

Yu, Y. G., Buss, G. R., and Maroof, M. A. S. 1996. Isolation of a superfamily of candidate disease-resistance genes in soybean based on a conserved nucleotide-binding site. Proc. Natl. Acad. Sci. USA 93:11751-11756.

Zhang, H.-B., Choi, S., Woo, S.-S., Li, Z., and Wing, R. A. 1996. Construction and characterization of two rice bacterial artificial chromosome libraries from the parents of a permanent recombinant inbred mapping population. Mol. Breed. 2:11-24. 